\title{
SETTING A BONUS-MALUS SCALE IN THE PRESENCE OF OTHER RATING FACTORS
}

\author{
GREG TAYLOR \\ Consultant, Tillinghast-Towers Perrin, \\ GPO Box 3279, \\ Sydney NSW 2001, AUSTRALIA \\ and \\ Professorial Associate, Centre for Actuarial Studies, \\ Faculty of Economics and Commerce, \\ University of Melbourne, \\ Parkville, Victoria 3052, AUSTRALIA
}

October, 1996

Revised April, 1997

\begin{abstract}
SUMMARY
The operation of a bonus-malus system, superimposed on a premium system involving a number of other rating variables, is considered. To the extent that good risks are rewarded in their base premiums, through the other rating variables, the size of the bonus they require for equity is reduced. This issue is discussed quantitatively, and a numerical example given.
\end{abstract}

\section{KEYWORDS}

Bonus-malus, experience rating.

\section{INTRODUCTION}

A system of bonus-malus (BM) calculates the premium applicable to particular contract as a base premium, adjusted by a quantity (the bonus or malus) which depends on previous claims experience.

Consider a BM system in which the BM has $\mathbf{J}$ possible values, called the BM levels. These may be labelled 1, 2, .., J, called the BM classes. The system is defined by the classes, levels, and the rules according to which claims experience is mapped to transitions between classes.

The collection of classes, together with their associated levels, will be referred to as the BM scale.

Over time, the portfolio will be distributed over the BM classes. In a typical BM system the distribution will ultimately stabilise. Because occupancy of each BM class 
is a function of claims experience, the individuals in the portfolio with low claim frequency parameters will tend to gravitate to the BM classes characterised by light claims experience. Conversely, for individuals with high claim frequencies.

The ultimate average claim frequency in each BM class defines the level to which that class is theoretically entitled. This, and related issues, have been dealt with many times in the literature. The two books of Lemaire $(1985,1995)$ provide a summary of a number of relevant matters.

It is common in such writings to assume that BM is the only means by which premiums are differentiated. In other words, all contracts are subject to the same base premium.

In practice, some portfolios, e.g. motor, are rated on a comparatively large (perhaps 10 or so) other variables. These will also differentiate individuals according to claim frequencies.

Consider the distribution of the portfolio over risk classes in the presence of these other rating variables. If they are used effectively by the premium system, then those $\mathrm{BM}$ classes with low average claim frequencies will tend to have low base premiums also.

In this event, the justifiable BM levels need to recognise the differentiation of underlying claim frequency by experience, but only to the extent that this differentiation is not already recognised within base premiums.

Subsequent sections of this paper examine the detail of this issue.

\section{NOTATION}

Let:

$\theta=$ vector of covariates (e.g. age, sex, etc.) with risk premium of an individual;

$\Lambda=$ an individual's true underlying risk premium.

It is asumed that, for given $\theta$, there is a distribution of values of $\Lambda$. Suppose that the pdf of $\Lambda$, conditioned on $\theta$, takes the form:

$$
f(\lambda \mid \theta)=g(\lambda \mid \mu(\theta))
$$

for some pdf $g($.$) and where$

$$
\mu(\theta)=E[\Lambda \mid \theta]
$$

The parameter $\theta$ will vary from one contract to antother, and hence so does $\mu(\theta)$. Let

$$
h(\mu)=\text { pdf of } \mu \text { over the whole portfolio. }
$$

Now introduce a BM system with classes $1,2, \ldots, \mathrm{J}$, and let

$\pi_{j}^{(t)}(\lambda)=$ probability that a policy owner with underlying risk premium $\lambda$ occupies $\mathrm{BM}$ class $j$ in the $\mathrm{t}$-th period since commencement of the system.

Note that 


$$
\sum_{j=1}^{J} \pi_{j}^{(t)}(\lambda)=1 \text { for each } t, \lambda .
$$

The system is initialised at $t=1$. It is assumed Markovian.

For most realistic BM systems, the vector $\left[\pi_{1}^{(t)}(\lambda), \ldots, \pi_{J}^{(t)}(\lambda)\right]$, representing the distribution of BM levels in period $t$ of risks characterised by $\lambda$, will approach a steady state with increasing $t$. It will be assumed here that such a steady state exists, and that convergence to it occurs over time. Let $\pi_{j}(\lambda)=$ the steady state value of $\pi_{j}^{(t)}(\lambda)$.

One can define the Bayesian posterior expectations:

$$
\begin{aligned}
& \lambda_{j}^{(t)}=\mathrm{E}[\Lambda \mid \mathrm{BM} \text { class in } \mathrm{t}-\mathrm{th} \text { period }=j], \\
& \mu_{j}^{(t)}=\mathrm{E}[\mu \mid \mathrm{BM} \text { class in } \mathrm{t}-\mathrm{th} \text { period }=j],
\end{aligned}
$$

and let $\lambda_{j}, \mu_{j}$ be the steady state versions of $\lambda_{j}^{(t)}, \mu_{j}^{(t)}$.

One way of viewing these quantities is as follows. The portfolio consists of two levels of heterogeneity:

- different risk classes defined by different $\mu(\theta)$; and

- within these different risk classes, different individuals characterised by their personal values of $\lambda$.

The quantities $\mu_{j}^{(t)}$ indicate the extent to which the BM system differentiates the risk classes over time. The quantities $\lambda_{j}^{(t)}$ indicates the extent to which the BM system differentiates individuals over time.

\section{SETTING THE BONUS-MALUS SCALE}

The Bayesian expectation $\lambda_{j}^{(t)}$ can be represented as:

$$
\lambda_{j}^{(t)}=\int \lambda p^{(t)}(\lambda, j) d \lambda / \int p^{(t)}(\lambda, j) d \lambda
$$

where $p($.$) will be used generically to denote a pdf and in this case p^{(t)}($.$) is a pdf in the$ $\mathrm{t}$-th interval.

Now the joint pdf in (3.1) can be expanded:

$$
\begin{aligned}
p^{(t)}(\lambda, j) & =\pi_{j}^{(t)}(\lambda) p^{(t)}(\lambda) \\
& =\pi_{j}^{(t)}(\lambda) \int g(\lambda \mid \mu) h(\mu) d \mu .
\end{aligned}
$$

By (3.1) and (3.2),

$$
\lambda_{j}^{(t)}=\frac{\int d \mu d \lambda \lambda \pi_{j}^{(t)}(\lambda) g(\lambda \mid \mu) h(\mu)}{\int d \mu d \lambda \pi_{j}^{(t)}(\lambda) g(\lambda \mid \mu) h(\mu)}
$$

Similarly, 


$$
\mu_{j}^{(t)}=\frac{\int d \mu d \lambda \mu \pi_{j}^{(t)}(\lambda) g(\lambda \mid \mu) h(\mu)}{\int d \mu d \lambda \pi_{j}^{(t)}(\lambda) g(\lambda \mid \mu) h(\mu)}
$$

Define

$$
r_{j}^{(t)}=\lambda_{j}^{(t)} / \mu_{j}^{(t)}
$$

As in Section 2, the absence of the time index indicates the steady state, i.e.

$$
r_{j}=\lambda_{j} / \mu_{j}
$$

To interpret $r_{j}^{(t)}$, first consider the degenerate case in which $h($.) concentrates all mass at a single value $\mu$. That is, the portfolio contains only one value of $\theta$; there is no variation of risk covariates, which in turn means that all policy owners are indistinguishable before the accumulation of claims experience. This is the case most commonly considered in the literature.

In this case (3.4) gives

$$
u_{j}^{(t)}=\mu
$$

hence (3.5) becomes

$$
r_{j}^{(t)}=\lambda_{j}^{(t)} / \mu
$$

The number $\lambda_{j}^{(t)}$, is effectively the Bayesian revision of $\mu$ taking into account the information that BM level is $j$ in the $\mathrm{t}$-th period. Thus $r_{j}^{(t)}$, is the factor by which the Bayesian revision adjusts the policy owners' prior expectation. Equivalently, $r_{j}^{(t)}$ is the factor by which $t$ years of experience revises the prior risk premium in BM class $j$.

The situation involving general $h($.$) is similar. However, in this case the composition$ of $\mathrm{BM}$ class $j$ with respect to the prior expectation $\mu(\theta)$ will change over time. For example, there will be a tendency for the contracts with the lowest priors to migrate to the $\mathrm{BM}$ class with lightest claims experience. Thus, $\mu_{j}^{(i)}$, tracks the average prior in BM level $j$ over time.

Despite this change, $r_{j}^{(t)}$, still denotes the factor by which experience revises the average prior risk premium in $\mathrm{BM}$ level $j$.

The relevance of this is as follows. The average prior $\mu_{j}^{(t)}$ is the average "standard premium rate" (i.e. the rate before recognition of experience) applicable to BM class $j$ in the $\mathrm{t}$-th year. Thus $100\left[r_{j}^{(t)}-1\right]$ is the BM percentage justified by experience in class $j$.

Suppose that $\mathrm{BM}$ class $\mathrm{K}$ receives these standard rates. Then the factor which can be justified as relating BM class $j$ to standard rates is $r_{j}^{(t)} / r_{K}^{(t)}$. These factors can be summarised in the vector

$$
r_{*}^{(t)}=r^{(t)} / r_{K}^{(t)}
$$

where $r^{(t)}$ is the vector with components $r_{j}^{(t)}$. 
The conclusion is that the maximum differentiation between premiums for different classes will be according to a factor

$$
\max _{j} r_{j} / \min _{j} r_{j}
$$

with $r_{j}$ defined by (3.6), i.e. a factor of

$$
\max _{j}\left(\lambda_{j} / \mu_{j}\right) / \min _{j}\left(\lambda_{j} / \mu_{j}\right)
$$

If the differentiation of priors $\mu_{j}$ over BM classes is left out of account, the differentiation of premiums wil be according to a factor of

$$
\max _{j} \lambda_{j} / \min _{j} \lambda_{j}
$$

which will usually be substantially larger than (3.11).

\section{NUMERICAL EXAMPLE}

A specifically structured portfolio of risks, subject to a particular BM system, has been simulated and values of $\lambda_{j}^{(t)}, \mu_{j}^{(t)}$ recorded.

The portfolio consists of 10 groups of individuals structured as follows.

TABLE 4.1

PORTFOLIO STRUCTURE

\begin{tabular}{cccc}
\hline Risk Group & $\begin{array}{c}\text { Mean cell average } \\
\text { claim frequency }\end{array}$ & $\begin{array}{c}\text { Coefficient of variation } \\
\text { of within-cell claim } \\
\text { frequency } \\
\boldsymbol{\%}\end{array}$ & $\begin{array}{c}\text { Proportion } \\
\text { of portfolio }\end{array}$ \\
\hline $\mathbf{\%}$ & 6.5 & 75 & $\%$ \\
$\mathbf{2}$ & 8.9 & 65 & 4.0 \\
$\mathbf{3}$ & 11.4 & 60 & 18.9 \\
$\mathbf{4}$ & 13.7 & 55 & 20.1 \\
$\mathbf{5}$ & 16.1 & 50 & 12.0 \\
$\mathbf{6}$ & 20.1 & 45 & 11.6 \\
$\mathbf{7}$ & 24.9 & 40 & 4.3 \\
$\mathbf{8}$ & 29.7 & 40 & 2.1 \\
$\mathbf{9}$ & 36.0 & 40 & 0.6 \\
\hline $\mathbf{1 0}$ & 50.5 & & 100 \\
\hline
\end{tabular}

This structure was obtained by constructing a multiplicative model of claim frequency according to a number of covariates (but excluding BM), and then counting the numbers of policies in bands of modelled claim frequency, $5-7.5 \%, 7.5 \%-10 \%$, etc.

The coefficient of variation of each band was chosen largely by informed guesswork, but subject to the criterion, again guesswork, that within-cell variance should increase in absolute terms with increasing frequency, but decrease in relative terms. 
Individuals within a particular risk group are sampled from a certain gamma distribution with the parameters set out in Table 4.1, as will be described later. There are 9 BM classes, of which Class 6 is the standard. A higher class number indicates a higher premium. The rules for transition between the classes are as follows.

TABLE 4.2

BM TRANSITION RULES

\begin{tabular}{ccccc}
\hline $\begin{array}{c}\text { Opening } \\
\text { Class }\end{array}$ & Closing class after a year if & \\
& 0 claims & I claim & 2 claims & $\begin{array}{c}\text { or more } \\
\text { claims }\end{array}$ \\
\hline $\mathbf{9}$ & 8 & 9 & 9 & 9 \\
$\mathbf{8}$ & 7 & 9 & 9 & 9 \\
$\mathbf{7}$ & 6 & 8 & 9 & 9 \\
$\mathbf{6}$ & 5 & 7 & 8 & 9 \\
$\mathbf{5}$ & 4 & 7 & 8 & 8 \\
$\mathbf{4}$ & 3 & 6 & 7 & 8 \\
$\mathbf{3}$ & 2 & 5 & 7 & 7 \\
$\mathbf{2}$ & 1 & 4 & 6 & 7 \\
$\mathbf{1}$ & 1 & 3 & 5 & -1 \\
\hline \hline
\end{tabular}

Appendix A gives the technical detail of the simulation

The claims experience of this portfolio is simulated over 30 years. At the beginning of year 1 all insureds are assumed to be in Class 6. The distribution appears to stabilise by about the end of Year 24. Consequently, the following results are averages over years 24 to 30 .

TABLE 4.3

Simulation Results

\begin{tabular}{ccccc}
\hline \hline $\begin{array}{c}\boldsymbol{B} \text { Class } \\
\boldsymbol{j}\end{array}$ & $\begin{array}{c}\text { Average } \\
\text { Proportion of } \\
\text { portfolio } \\
\%\end{array}$ & $\begin{array}{c}\text { True claim } \\
\text { frequency } \lambda_{j}\end{array}$ & $\begin{array}{c}\text { Cell claim } \\
\text { frequency } \mu_{j}\end{array}$ & $\begin{array}{c}\text { Ratio: true/cell } \\
\text { claim frequency } \\
\boldsymbol{r}_{j}\end{array}$ \\
\hline $\mathbf{9}$ & 1 & $\%$ & $\%$ & $\%$ \\
$\mathbf{8}$ & 1 & 46 & 26 & 175 \\
$\mathbf{7}$ & 2 & 38 & 24 & 156 \\
$\mathbf{6}$ & 3 & 32 & 22 & 145 \\
$\mathbf{5}$ & 4 & 30 & 22 & 139 \\
$\mathbf{4}$ & 4 & 23 & 20 & 111 \\
$\mathbf{3}$ & 10 & 21 & 19 & 103 \\
$\mathbf{2}$ & 9 & 18 & 17 & 102 \\
$\mathbf{1}$ & 66 & 17 & 14 & 85 \\
\hline \hline
\end{tabular}

The table shows that, if base premiums reflect cell claim frequencies accurately, the BM scale should vary by a maximum factor of about 2 [cf (3.11)]. If the variation of the base premiums were left out of account, the BM scale would vary by a factor of nearly 4 [cf (3.12)]. The BM scale justified by the middle columns of Table 4.3 in the case $K=6$ is as follows. 
TABLE 4.4

PREMIUMS FOR BM Classes

\begin{tabular}{ccc}
\hline $\begin{array}{c}\text { BM Class } \\
\boldsymbol{j}\end{array}$ & \multicolumn{2}{c}{$\begin{array}{c}\text { Premium as } \% \\
\text { of standard } \\
\text { recognising covariates }\end{array}$} \\
\hline $\mathbf{9}$ & 126 & 152 \\
$\mathbf{8}$ & 112 & 125 \\
$\mathbf{7}$ & 104 & 107 \\
$\mathbf{6}$ & 100 & 100 \\
$\mathbf{5}$ & 83 & 77 \\
$\mathbf{4}$ & 80 & 71 \\
$\mathbf{3}$ & 74 & 59 \\
$\mathbf{1}$ & 73 & 57 \\
\hline
\end{tabular}

If these premiums had been computed from the column of $\lambda_{j}$ in Table 4.3 , ignoring the effect of the covariates, quite different, and misleading, results would have been obtained, as shown in the final column of Table 4.4.

It is of interest to examine how results of this type vary as the BM system varies. Consider the case in which Table 4.2 is replaced by a simple set of rules which provide for:

- 1 step forward for each claim-free year;

- 4 steps back for each claim.

This is much more severe than Table 4.2 which is largely a 1-forward/2-back set of rules.

The new system replaces Table 4.4 by Table 4.5 .

TABLE 4.5

Premiums for BM Classes IN MORE SEVERE SySTEM

\begin{tabular}{ccc}
\hline $\boldsymbol{B} M$ Class & \multicolumn{3}{c}{$\begin{array}{c}\text { Premium as } \% \\
\text { of standard }\end{array}$} \\
$j$ & recognising covariates & ignoring covariates \\
\hline $\mathbf{9}$ & 123 & 150 \\
$\mathbf{8}$ & 113 & 125 \\
$\mathbf{7}$ & 104 & 109 \\
$\mathbf{6}$ & 100 & 100 \\
$\mathbf{5}$ & 92 & 83 \\
$\mathbf{4}$ & 91 & 81 \\
$\mathbf{2}$ & 86 & 75 \\
$\mathbf{1}$ & 83 & 72 \\
\hline
\end{tabular}

If the ratio of the two columns in Table 4.5 is regarded as an "error ratio", measuring the error in ignoring covariates, the following comparison is noteworthy. 
TABLE 4.6

ERROR RATIOS FOR DIFFERENT BM SYSTEMS

\begin{tabular}{ccc}
\hline $\begin{array}{c}\text { BM Class } \\
\boldsymbol{j}\end{array}$ & $\begin{array}{c}\text { Error Ratio } \\
\text { Original BM system } \\
\text { (Table 4.4) } \\
\mathbf{\%}\end{array}$ & $\begin{array}{c}\text { Severe BM system } \\
\text { (Table 4.5) } \\
\%\end{array}$ \\
\hline $\mathbf{9}$ & 121 & 122 \\
$\mathbf{8}$ & 112 & 111 \\
$\mathbf{7}$ & 103 & 105 \\
$\mathbf{6}$ & 100 & 100 \\
$\mathbf{5}$ & 93 & 81 \\
$\mathbf{4}$ & 89 & 88 \\
$\mathbf{3}$ & 79 & 86 \\
$\mathbf{1}$ & 78 & 75 \\
\hline
\end{tabular}

Although the BM systems are very different, and so are the levels of bonus justified by them, there is a good deal of similarity between their error ratios.

\section{APPENDIX A}

TECHNICAL DETAIL OF SIMULATION

\section{Individual claim frequency}

Consider an individual in BM class $j$ with $\mu_{j}^{(1)}$ given by Table 4.1 . Let $w_{j}$ be the associated coefficient of variation in Table 4.1. The value of $\Lambda$ for this individual is assumed to be

$$
\Lambda=\mu_{j}^{(1)}\left[1+w_{j}^{(X / 2-1)}\right]
$$

where $X \sim \chi_{2}^{2}$.

Since $\chi_{2}^{2}$ is gamma with mean 2 and standard deviation 2, (A.1) gives $E[\Lambda]=\mu_{j}^{(1)}$, s.d. $[\Lambda]=w_{j} \mu_{j}^{(1)}$, as required.

Values of $X$ are simulated as:

$$
X=X_{1}^{2}+X_{2}^{2}
$$

where $X_{1}, X_{2}$ are independent, and

$$
X_{i} \approx N(0,1), i=1,2 \text {. }
$$

\section{Claim inter-arrival times}

For the individual discussed in Appendix A.1, it is assumed that the number of claims in a year is distributed Poisson $(\Lambda)$. Hence inter-arrival times are exponentially distributed with mean $1 / \Lambda$. These inter-arrival times have been simulated as:

$$
T=-[\log (1-U)] / \Lambda \text {, }
$$


where $U$ is uniform $[0,1]$.

Note that (A.4) is equivalent to:

$$
U=1-\exp (-\Lambda T)
$$

from which exponentiality of $\mathrm{T}$ is easily proved.

\section{REFERENCES}

Lemaire, J. (1985) Automobile insurance: actuarial models. Kluwer - Nijhoff Publishing, Boston.

LEMAIRE, J. (1995) Bonus-malus systems in automobile insurance. Kluwer - Academic Publishers, Boston. 